\title{
Assessing Systematic Errors in Simulation Studies of Mu2e
}

\section{Introduction to Mu2e Simulations \\ About Mu2e}

The Muon-to-Electron Conversion Experiment (Mu2e) aims to measure the ratio of the rate of coherent conversion of muons to electrons in the field of an atomic nucleus relative (a charged lepton flavor violation decay) to the rate of ordinary muon capture on the nucleus, $R_{\mu e}$ :

$$
R_{\mu e}=\frac{\mu^{-}+A(Z, N) \rightarrow e^{-}+A(Z, N)}{\mu^{-}+A(Z, N) \rightarrow v_{\mu}+A(Z-1, N)}
$$

Currently, the Standard Model provides an experimental limit on the order of $<10^{-50} R_{\mu e}$, while Mu2e is projected to measure $R_{\mu e}$ with a sensitivity on the order of $\sim 10^{-17}$, a measurement that would be sensitive to New Physics [1]

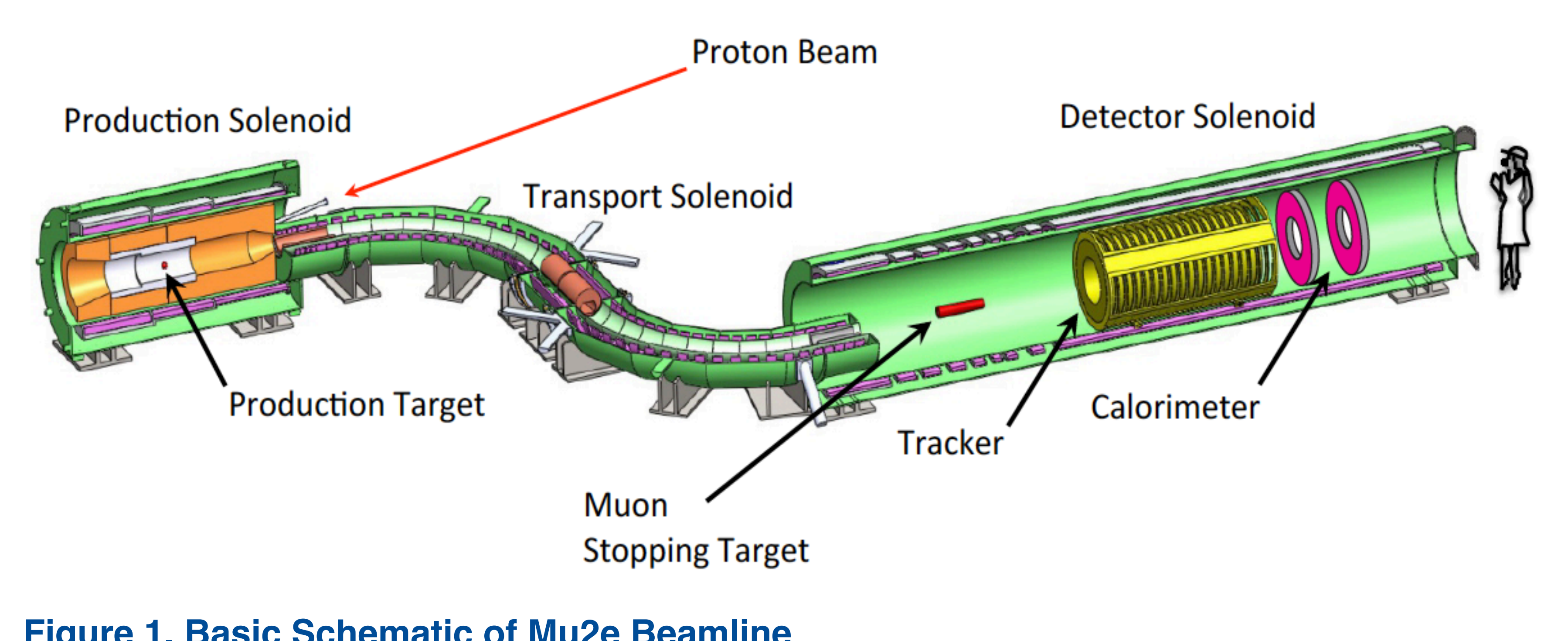

The Mu2e beamline is divided into three main parts: the production solenoid, the transport solenoid, and the detector solenoid. $8 \mathrm{GeV}$ protons produced by the Fermilab Booster strike a tungsten production target to produce pions that are conducted down the beamline, eventually decaying to muons of the desired energy via a magnetic field gradient. Pion and muon production is essential to the production of electrons at $\sim 105 \mathrm{MeV} / \mathrm{c}$ to be detected in the Mu2e detector. The present analyses is primarily concerned with simulation studies of particle production at the production solenoid.

\section{G4beamline vs. MARS15 Simulations}

G4beamline (G4BL) and MARS15 are two Monte Carlo simulators used for simulation of the Mu2e beamline. Both models are independently developed from one another, and both simulate particle transport and interaction with matter on the nucleus to produce secondary particles.

G4beamline, based on the GEANT4 toolkit [2], is primarily concerned with simulation of beamline and resulting matter interactions; MARS15 is Fortran/C++ code developed to simulate three-dimensional hadronic and electromagnetic cascades and modeling of heavy ion, muon and low energy neutron-photon transport [3]. By simulating identical phenomena in the experiment with two independent simulation programs, comparisons of their respective data products can be used to assess systematic errors in Mu2e.

\section{Methods \& Results}

In G4beamline, two virtual detectors of definite length were placed $1.0 \mathrm{~cm}$ downstream and upstream of the proton target. The detectors were established to be sensitive to charged muons and pions. An additional detector was placed directly upstream of the collimator at the entrance of the transport solenoid. In MARS15, planar virtual detectors were installed at the same coordinates as those specified by the geometry in G4beamline.

$1,000,000$ events were run in each program, and normalized frequency histograms were produced for each particle at each detector using G4BL and MARS. These results are compiled below.
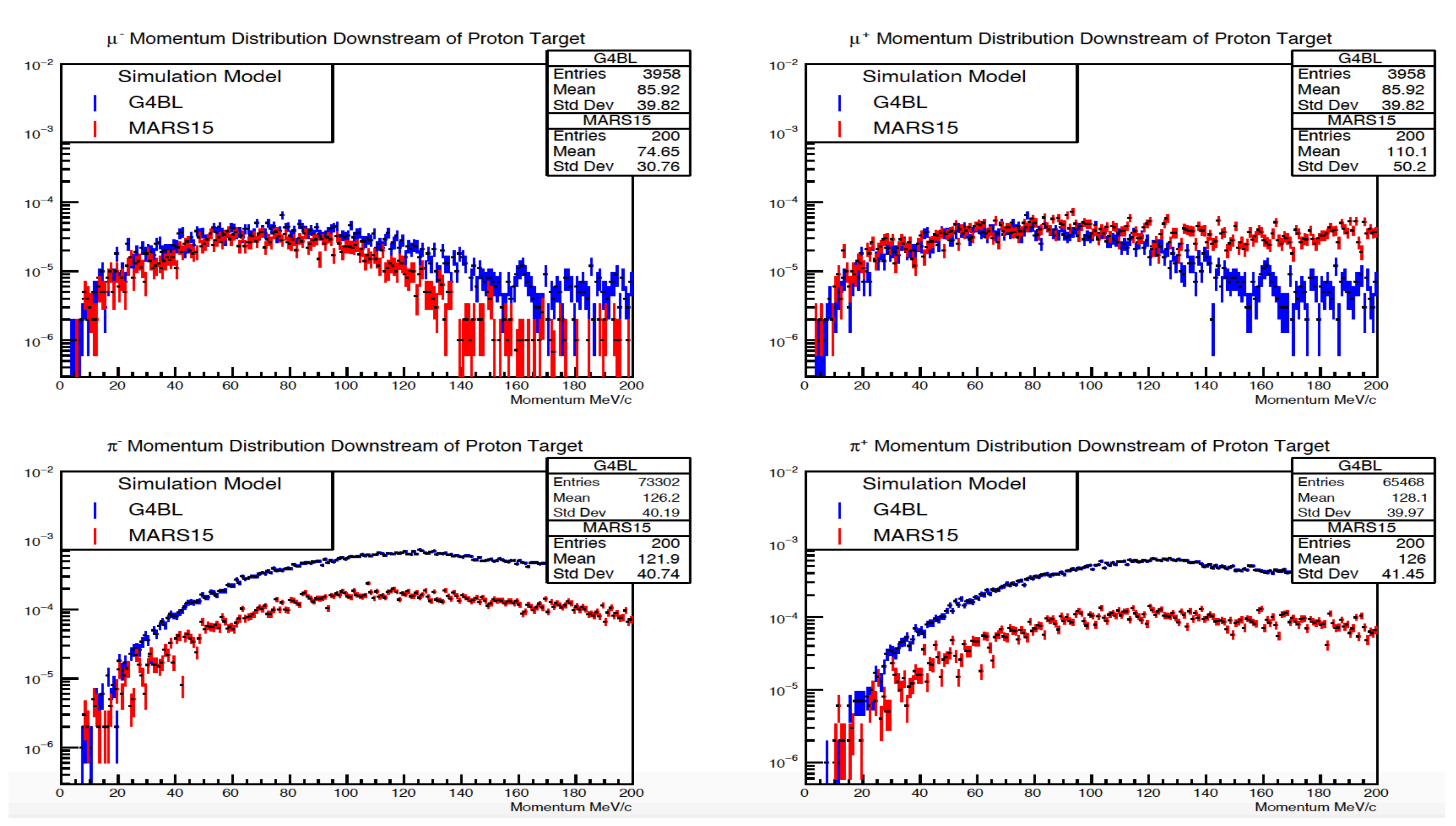

Figure 2. Momentum Distributions Downst
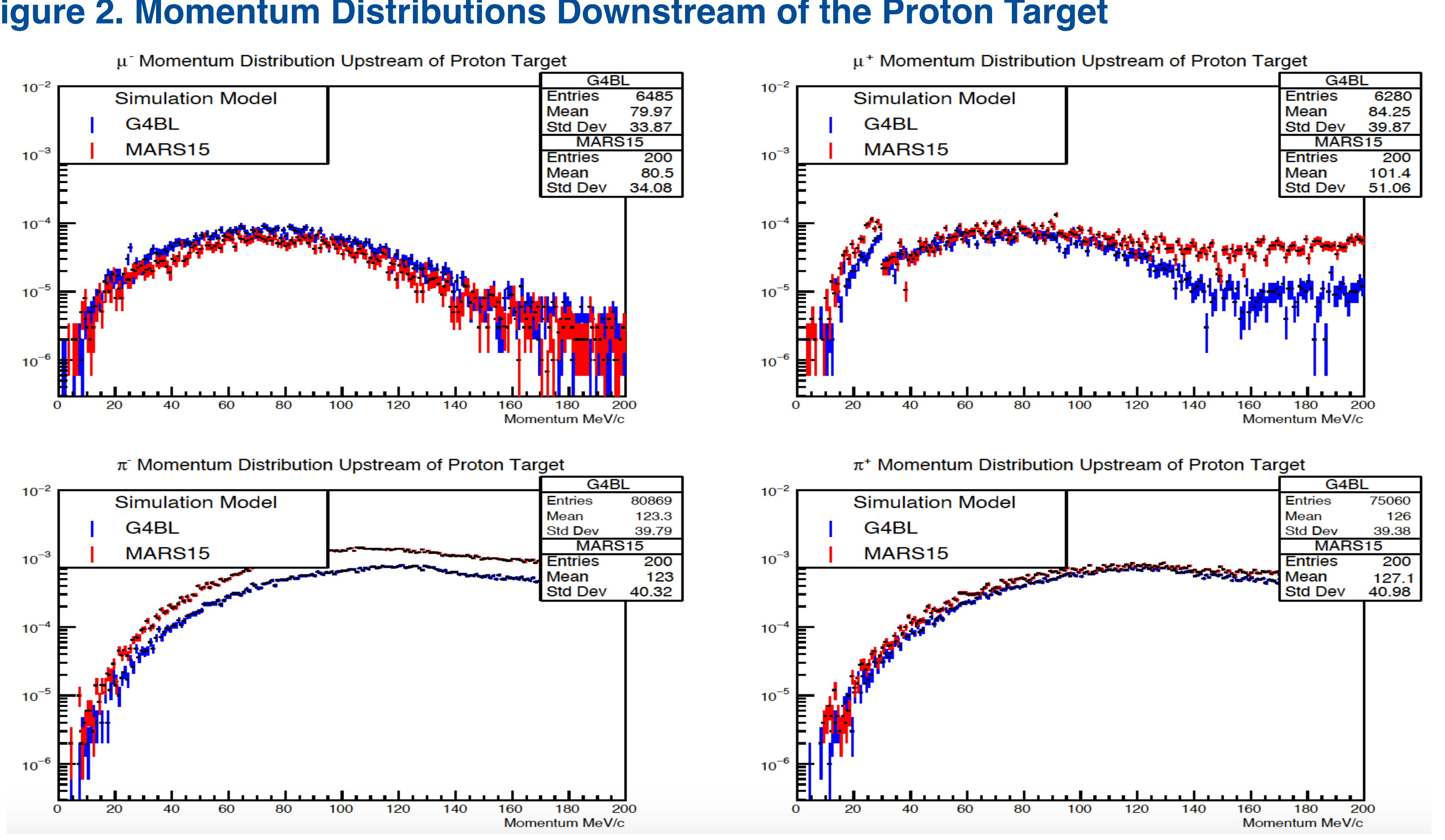

Figure 3. Momentum Distributions Upstream of the Proton Target
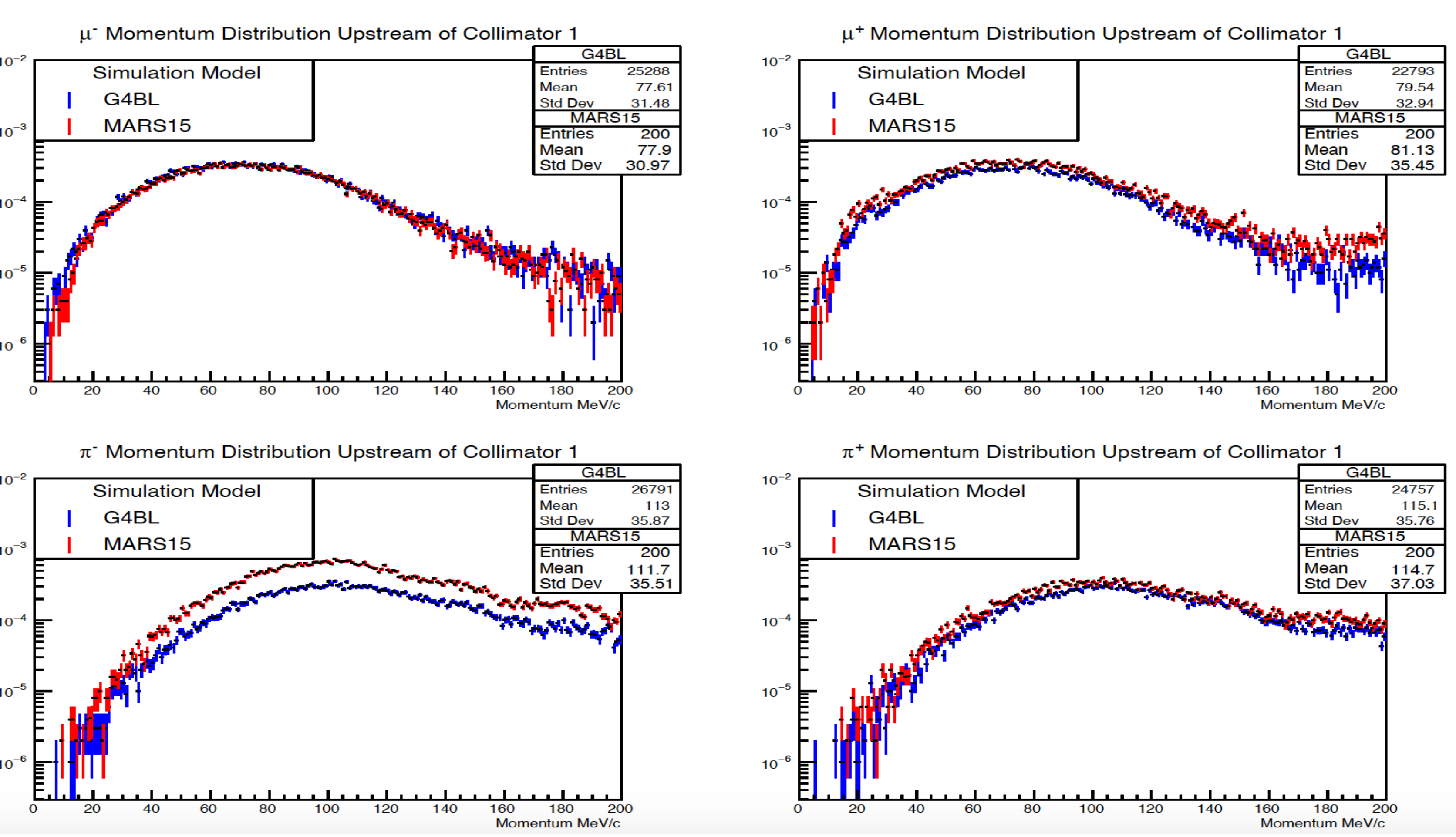

Figure 4. Momentum Distributions Upstream of the First Collimator

\section{Discussion}

Of importance to the simulation of the Mu2e beamline is production of $\mu^{-}$with momenta of $\sim 45 \mathrm{MeV} / \mathrm{c}$ at the entrance of the first collimator. The percent error of the bin centers at this momentum range (see Figure 4 ) is $\sim 6.68 \%$, and within this error it is impossible to distinguish the curves. It can be concluded, then, that the systematic error for $\mu^{-}$flux is $\sim 7 \%$, and both MARS15 and G4beamline can be used interchangeably for simulation of $\mu^{-}$production.

There are clear model dependencies between MARS15 and G4BL, however. In particular, $\pi^{-}$production is higher in G4BL than in MARS when looking downstream of the proton target, but the trend reverses when looking upstream of the proton target. Still, this does not appear to affect the $\mu^{-}$flux at the collimator, as the differences between the codes levels out.

The $\mu^{+}$fluxes at the first collimator do show a significant systematic error of $\sim 20-30 \%$ that requires further improvements in the simulations for any potential work on $\mu^{+}$production.

\section{References \& Acknowledgements}

[1] Mu2e Collaboration. (2014). Mu2e Technical Design Report. Batavia, IL: Fermi National Accelerator Laboratory.

[2] Roberts, T. (2018). G4beamlineUser's Guide (3.06 ed.). Muons.

[3] Mokhov, N., \& James, C. (2016). The MARS Code System User's Guide, Version 15. Batavia, IL: Fermi National Accelerator Laboratory.

I would like to give thanks to my mentor, Dr. Vitaly Pronskikh, whose help was indispensable for completing this work.

This work was supported in part by the U.S. Department of Energy, Office of Science, Office of Workforce Development for Teachers and Scientists (WDTS) Repor the Science Undergraduate Laboratory Interns 